\title{
WEIGHTED INEQUALITIES FOR QUASI-MONOTONE FUNCTIONS
}

\author{
LECH MALIGRANDA \\ Dedicated to the memory of my father, Kazimierz Maligranda 1917-93
}

\begin{abstract}
The purpose of this paper is to give characterizations of weights for which reverse inequalities of Hölder type for quasi-monotone functions are satisfied. Our inequalities with general weights and with sharp constants complement the results of $[\mathbf{2}, \mathbf{8}, \mathbf{9}]$ and $[\mathbf{1 6}, \mathbf{1 7}]$ for the values of parameters $p$ and $q$ with $1 \leqslant p$ $\leqslant q<\infty$.
\end{abstract}

\section{Introduction}

We consider positive quasi-monotone functions $f$ on $(0, \infty)$. We shall write $f \in Q_{\alpha}$ when $x^{-\alpha} f(x)$ is decreasing and $f \in Q^{\alpha}$ when $x^{-\alpha} f(x)$ is increasing. The quasi-monotone function $f$ satisfies both of the monotonicity conditions $Q_{\alpha_{1}}$ and $Q^{\alpha_{0}}$ for some $\alpha_{0}, \alpha_{1}$ with $-\infty<\alpha_{0}<\alpha_{1}<\infty$, that is,

$$
0 \leqslant f(s) \leqslant \max \left((s / t)^{\alpha_{0}},(s / t)^{\alpha_{1}}\right) f(t) \text { for all } s, t>0 .
$$

A class of functions of this type was studied some years ago by Zygmund (see, for example, papers by Chen [6], Chan [5], Persson [13] or Bingham, Goldie and Teugels $[4$, p. 123]).

The purpose of this paper is to find conditions on weight functions $u$ and $v$ such that the inequality

$$
\left(\int_{0}^{\infty} f(x)^{q} u(x) d x\right)^{1 / q} \leqslant C\left(\int_{0}^{\infty} f(x)^{p} v(x) d x\right)^{1 / p}
$$

holds, for any positive function $f$ from the class $Q^{\alpha_{0}} \cap Q_{\alpha_{1}}$. Our main aim is to prove such inequalities with the best constants. Surprisingly enough, this was possible for all parameters in the range $1 \leqslant p \leqslant q<\infty$. Some results of this type were proved earlier by Lorentz and Hunt (see [15]), Bergh [1], Bergh, Burenkov and Persson [2], Stepanov [16], Heinig and Stepanov [9], Heinig and Maligranda [8], Pečarić and Persson [12] and recently by Gol'dman, Heinig and Stepanov [7].

Positive functions considered throughout the paper are non-negative and not identically zero on $(0, \infty)$. We shall write $0 \leqslant f \downarrow$ to mean that the function $f$ is positive and decreasing (where decreasing means non-increasing); for such an $f$ we define $f^{-1}(t)=\inf \{s>0: f(s)<t\}$, inf $\varnothing=\infty$. Similarly, $0 \leqslant f \uparrow$ means that $f$ is positive and increasing (increasing means non-decreasing); for such an $f$ we define $f^{-1}(t)=$ $\inf \{s>0: f(s)>t\}, \inf \varnothing=\infty$.

Weight functions are locally integrable positive functions on $(0, \infty)$, usually

Received 20 February 1995; revised 17 November 1995.

1991 Mathematics Subject Classification 26D15.

J. London Math. Soc. (2) 57 (1998) 363-370 
denoted by $u, v, w$, and $1_{E}$ denotes the characteristic function of the set $E$. The $L_{p, w^{-}}$ norm is the functional

$$
\|f\|_{p, w}=\left(\int_{0}^{\infty} f(x)^{p} w(x) d x\right)^{1 / p}
$$

Inequalities such as that in $\left(^{*}\right)$ are interpreted as meaning that if the right-hand side is finite, then so is the left-hand side, and the inequality holds.

\section{The main result}

The following lemma will be useful in the proof of our main result, Theorem 1.

Lemma 1. Let $w$ be a weight function and let $r$ and $\gamma$ be such that $0<r<\infty, 0<$ $\gamma \leqslant 1$

If $0 \leqslant \phi \downarrow$, then

and

$$
\left(\int_{0}^{\infty} \phi^{-1}(x)^{1 / \gamma} w(x) d x\right)^{\gamma} \leqslant \int_{0}^{\infty}\left[\int_{0}^{\phi(y)} w(x) d x\right]^{\gamma} d y
$$

$$
\int_{\phi^{-1}(x)}^{\infty} \phi(y)^{r} d y=\int_{0}^{x} \phi^{-1}(s) d\left(s^{r}\right)-x^{r} \phi^{-1}(x)
$$

If $0 \leqslant \phi \uparrow$, then

$$
\left(\int_{0}^{\infty} \phi^{-1}(x)^{1 / \gamma} w(x) d x\right)^{\gamma} \leqslant \int_{0}^{\infty}\left[\int_{\phi(y)}^{\infty} w(x) d x\right]^{\gamma} d y .
$$

For $\gamma=1$ we have equalities in (1) and (3).

Proof. First we show that for the positive decreasing function $\phi$ on $(0, \infty)$ we have

$$
\phi^{-1}(x) \leqslant \int_{0}^{\infty} 1_{[0, \phi(y)]}(x) d y .
$$

In fact, let $\phi^{-1}\left(0^{+}\right)=\infty$ and $\phi^{-1}(\infty)=0$. If $0<y<\phi^{-1}(x)$, then $x \leqslant \phi(y)$ and we have

$$
\phi^{-1}(x)=\int_{0}^{\phi^{-1}(x)} d y=\int_{0}^{\infty} 1_{\left[0, \phi^{-1}(x)\right)}(y) d y \leqslant \int_{0}^{\infty} 1_{[0, \phi(y)]}(x) d y .
$$

In the case when $\phi^{-1}\left(0^{+}\right)<\infty$ or $\phi^{-1}(\infty)>0$ we can modify this argument to prove that the inequality $(* *)$ is true also in these cases.

Then, by using the Minkowski inequality with the $L_{1 / \gamma, w}$-norm, we find that

$$
\begin{aligned}
\left(\int_{0}^{\infty} \phi^{-1}(x)^{1 / \gamma} w(x) d x\right)^{\gamma} & =\left\|\phi^{-1}(x)\right\|_{1 / \gamma, w} \leqslant\left\|\int_{0}^{\infty} 1_{[0, \phi(y)]}(x) d y\right\|_{1 / \gamma, w} \\
& \leqslant \int_{0}^{\infty}\left\|1_{[0, \phi(y)]}(x)\right\|_{1 / \gamma, w} d y \\
& =\int_{0}^{\infty}\left[\int_{0}^{\phi(y)} w(x) d x\right]^{\gamma} d y
\end{aligned}
$$

and the inequality (1) is proved.

Moreover, it is geometrically clear that

$$
\int_{0}^{x^{r}} \phi^{-1}\left(t^{1 / r}\right) d t=x^{r} \phi^{-1}(x)+\int_{\phi^{-1}(x)}^{\infty} \phi(y)^{r} d y .
$$


Combining this with the obvious equality $\int_{0}^{x^{r}} \phi^{-1}\left(t^{1 / r}\right) d t=\int_{0}^{x} \phi^{-1}(s) d\left(s^{r}\right)$ we obtain proof of equality (2).

To prove inequality (3) for increasing $\phi$, we first show that the following inequality holds:

$$
\phi^{-1}(x) \leqslant \int_{0}^{\infty} 1_{[\phi(y), \infty)}(x) d y .
$$

Let $\phi^{-1}\left(0^{+}\right)=0$ and $\phi^{-1}(\infty)=\infty$. If $0<y<\phi^{-1}(x)$, then $\phi(y) \leqslant x$ and we have

$$
\phi^{-1}(x)=\int_{0}^{\phi^{-1}(x)} d y=\int_{0}^{\infty} 1_{\left[0, \phi^{-1}(x)\right)}(y) d y \leqslant \int_{0}^{\infty} 1_{[\phi(y), \infty)}(x) d y .
$$

The proof in the cases when $\phi^{-1}\left(0^{+}\right)>0$ or $\phi^{-1}(\infty)<\infty$ can be carried out by making a minor modification.

Then, again by using the Minkowski inequality with the $L_{1 / \gamma, w}$-norm, we find that

$$
\begin{aligned}
\left(\int_{0}^{\infty} \phi^{-1}(x)^{1 / \gamma} w(x) d x\right)^{\gamma} & =\left\|\phi^{-1}(x)\right\|_{1 / \gamma, w} \leqslant\left\|\int_{0}^{\infty} 1_{[\phi(y), \infty)}(x) d y\right\|_{1 / \gamma, w} \\
& \leqslant \int_{0}^{\infty}\left\|1_{[\phi(y), \infty)}(x)\right\|_{1 / \gamma, w} d y \\
& =\int_{0}^{\infty}\left[\int_{\phi(y)}^{\infty} w(x) d x\right]^{\gamma} d y,
\end{aligned}
$$

and inequality (3) is proved.

If $\gamma=1$, then the set $\left\{(x, y\}: y=\phi(x)\right.$ or $\left.x=\phi^{-1}(y)\right\}$, as the sum of the graphs of $\phi(x)$ and $\phi^{-1}(y)$, has two-dimensional Lebesgue measure zero (cf. [18]), and by Fubini's theorem we have equalities.

REMARK 1. If $\phi$ is an increasing function and $\phi\left(0^{+}\right)>0$, then inequality (3) follows from (1). We simply use inequality (1) on the decreasing function $\psi(x)=$ $1 / \phi(x)$ with weight $w_{1}(x)=w(1 / x) / x^{2}$ and change variable $x$ to $1 / x$.

REMARK 2. Using Lemma 1 we can prove the following results.

If $0<p \leqslant q<\infty$ and $-\infty<\alpha_{0}<\alpha_{1}<\infty$, then

$$
\sup _{0 \leqslant f \in Q_{\alpha_{1}}} \frac{\|f\|_{q, u}}{\|f\|_{p, v}}=\sup _{t>0}\left(\int_{0}^{t} x^{q \alpha_{1}} u(x) d x\right)^{1 / q}\left(\int_{0}^{t} x^{p \alpha_{1}} v(x) d x\right)^{-1 / p}
$$

and

$$
\sup _{0 \leqslant f \in Q^{\alpha_{0}}} \frac{\|f\|_{q, u}}{\|f\|_{p, v}}=\sup _{t>0}\left(\int_{t}^{\infty} x^{q \alpha_{0}} u(x) d x\right)^{1 / q}\left(\int_{t}^{\infty} x^{p \alpha_{0}} v(x) d x\right)^{-1 / p} .
$$

The equivalence (not equality) results for the classes $Q_{0}$ and $Q^{0}$ were proved by Sawyer [14], Stepanov [16] and Heinig and Stepanov [9]. Of course, it is enough to prove such results for $Q_{0}$ and $Q^{0}$ and then, by change of weights, to extend them to $Q_{\alpha_{1}}$ and $Q^{\alpha_{0}}$. A different set of equality proofs was given by Heinig and Maligranda $[8]$.

It can be proved that if $0<p \leqslant q<\infty, \alpha_{0}<\alpha<\alpha_{1}$ and $0 \leqslant f \in Q^{\alpha_{0}} \cap Q_{\alpha_{1}}$, then the inequality

$$
\left(\int_{0}^{\infty}\left(x^{-\alpha} f(x)\right)^{q} \frac{d x}{x}\right)^{1 / q} \leqslant C\left(\int_{0}^{\infty}\left(x^{-\alpha} f(x)\right)^{p} \frac{d x}{x}\right)^{1 / p}
$$

holds with the sharp constant $C=p^{1 / p} q^{-1 / q}\left(\left(\alpha_{1}-\alpha\right)^{-1}+\left(\alpha-\alpha_{0}\right)^{-1}\right)^{1 / q-1 / p}$. This result in the case when $\alpha_{0}=0$ and $\alpha_{1}=1$ was stated in [3] as the exact constant for the 
imbedding of real interpolation spaces $\left(A_{0}, A_{1}\right)_{\alpha, p} \subset\left(A_{0}, A_{1}\right)_{\alpha, q}$, and it was proved by Bergh [1] who used a symmetrization argument. Another proof of this result was given by Bergh, Burenkov and Persson [2]. Recently, with the same technique, Pečarić-Persson [12] proved the result for functions satisfying $0 \leqslant f(s) \leqslant$ $C \max \left((s / t)^{\alpha_{0}},(s / t)^{\alpha_{1}}\right) f(t)$ for all $s, t>0$, where $C \geqslant 1$.

The above result for power weights motivated us to give necessary and sufficient conditions on weights $u$ and $v$ such that the inequality $(*)$ holds for functions $f$ from the classes $Q^{\alpha_{0}} \cap Q_{\alpha_{1}}$. We shall prove this with good control of the constants.

TheOREM 1. Let $1 \leqslant p \leqslant q<\infty$ and $-\infty<\alpha_{0}<\alpha_{1}<\infty$. Then

$$
\left(\int_{0}^{\infty} f(x)^{q} u(x) d x\right)^{1 / q} \leqslant C\left(\int_{0}^{\infty} f(x)^{p} v(x) d x\right)^{1 / p} \quad \forall 0 \leqslant f \in Q^{\alpha_{0}} \cap Q_{\alpha_{1}}
$$

holds if and only if

$$
\begin{aligned}
D= & \sup _{t>0}\left[\left(\int_{0}^{t} x^{q \alpha_{1}} u(x) d x+t^{q\left(\alpha_{1}-\alpha_{0}\right)} \int_{t}^{\infty} x^{q \alpha_{0}} u(x) d x\right)^{1 / q}\right. \\
& \left.\times\left(\int_{0}^{t} x^{p \alpha_{1}} v(x) d x+t^{p\left(\alpha_{1}-\alpha_{0}\right)} \int_{t}^{\infty} x^{p \alpha_{0}} v(x) d x\right)^{-1 / p}\right]<\infty .
\end{aligned}
$$

Moreover, $D \leqslant C \leqslant 2 D$.

Proof. (4) $\Rightarrow(5)$. Take $f(x)=f_{t}(x)=\min \left(x^{\alpha_{1}}, t^{\alpha_{1}-\alpha_{0}} x^{\alpha_{0}}\right)$ for $t>0$ in (4). From this it follows that $D \leqslant C$.

Moreover, if $D<\infty$ and $\int_{0}^{\infty} x^{p \alpha_{1}} v(x) d x<\infty$, then

$$
t^{p\left(\alpha_{1}-\alpha_{0}\right)} \int_{t}^{\infty} x^{p \alpha_{0}} v(x) d x \leqslant \int_{t}^{\infty} x^{p \alpha_{1}} v(x) d x \longrightarrow 0 \quad \text { as } t \longrightarrow \infty
$$

and thus, according to (5), $\int_{0}^{\infty} x^{q \alpha_{1}} u(x) d x<\infty$. Hence

$$
t^{q\left(\alpha_{1}-\alpha_{0}\right)} \int_{t}^{\infty} x^{q \alpha_{0}} u(x) d x \leqslant \int_{t}^{\infty} x^{q \alpha_{1}} u(x) d x \longrightarrow 0 \quad \text { as } t \longrightarrow \infty .
$$

Therefore

$$
\left(\int_{0}^{\infty} x^{q \alpha_{1}} u(x) d x\right)^{1 / q}\left(\int_{0}^{\infty} x^{p \alpha_{1}} v(x) d x\right)^{-1 / p} \leqslant D .
$$

$(5) \Rightarrow(4)$. First, let us derive some representation formulas for our functions.

If $0 \leqslant f \in Q^{\alpha_{0}} \cap Q_{\alpha_{1}}$, then the function $h$ defined by

$$
h(x)=x^{-\alpha_{0} /\left(\alpha_{1}-\alpha_{0}\right)} f\left(x^{1 /\left(\alpha_{1}-\alpha_{0}\right)}\right)
$$

belongs to $Q^{0} \cap Q_{1}$. It is known that the smallest concave majorant $k$ of $h$, defined by

$$
k(x)=\inf _{t>0}\left(1+\frac{x}{t}\right) h(t) \text { for } x>0,
$$

satisfies the inequalities $h(x) \leqslant k(x) \leqslant 2 h(x)$ for all $x>0$ (see, for example, [3, Lemma 5.4.3] or [10, Lemma 14.1]); and also that $k$, as a concave function, has the representation

$$
k(x)=\alpha+\int_{0}^{x} m(s) d s, \quad \text { for } 0 \leqslant m \downarrow,
$$

with $\alpha=\lim _{x \rightarrow 0^{+}} k(x)$ and $m(s)=k^{\prime}(s)$, where $k^{\prime}(s)$ is the right-derivative, which is a positive right-continuous decreasing function. 
The concave function $k(x)$ has a left derivative at all points and a right derivative at all points: the derivatives are decreasing and coincide except on the set of their (common) discontinuities, which is countable. Thus $k(x)$ is differentiable almost everywhere with a decreasing derivative which also gives the above representation.

The function $g(x)=x^{\alpha_{0}} k\left(x^{\alpha_{1}-\alpha_{0}}\right)$ belongs to $Q^{\alpha_{0}} \cap Q_{\alpha_{1}}$ and has the representation

$$
g(x)=c_{0} x^{\alpha_{0}}+x^{\alpha_{0}} \int_{0}^{x^{\alpha_{1}-\alpha_{0}}} m(s) d s, \text { for } 0 \leqslant m \downarrow, \quad \text { with } c_{0}=\lim _{x \rightarrow 0^{+}} x^{-\alpha_{0}} g(x) .
$$

Since $k$ and $h$ are equivalent we conclude that $f$ and $g$ are equivalent. Thus it is enough to prove our result for a function $g$ with the representation (6).

We can now prove the implication $(5) \Rightarrow(4)$. Let $\int_{0}^{\infty} g(x)^{p} v(x) d x<\infty$ and $D<\infty$, that is, for every $t>0$ suppose that

$$
\left\{\int_{0}^{\infty}\left[\min \left(x^{\alpha_{1}}, t^{\alpha_{1}-\alpha_{0}} x^{\alpha_{0}}\right)\right]^{q} u(x) d x\right\}^{p / q} \leqslant D^{p} \int_{0}^{\infty}\left[\min \left(x^{\alpha_{1}}, t^{\alpha_{1}-\alpha_{0}} x^{\alpha_{0}}\right)\right]^{p} v(x) d x .
$$

We put $t=\phi(y)$, where $\phi$ is a positive decreasing function which will be chosen later on, and integrate from 0 to $\infty$ with respect to $y$ to obtain

$$
\begin{aligned}
\int_{0}^{\infty}\left\{\int_{0}^{\infty}\left[\min \left(x^{\alpha_{1}}, \phi(y)^{\alpha_{1}-\alpha_{0}} x^{\alpha_{0}}\right)\right]^{q} u(x) d x\right\}^{p / q} d y \\
\leqslant D^{p} \int_{0}^{\infty}\left\{\int_{0}^{\infty}\left[\min \left(x^{\alpha_{1}}, \phi(y)^{\alpha_{1}-\alpha_{0}} x^{\alpha_{0}}\right)\right]^{p} v(x) d x\right\} d y .
\end{aligned}
$$

By using the Minkowski inequality with the $L_{q / p, u}$-norm we find that

$$
\begin{aligned}
\int_{0}^{\infty}\left[\int_{0}^{\infty}(\right. & \left.\left.\min \left(x^{\alpha_{1}}, \phi(y)^{\alpha_{1}-\alpha_{0}} x^{\alpha_{0}}\right)\right)^{q} u(x) d x\right]^{p / q} d y \\
& =\int_{0}^{\infty}\left\|\left(\min \left(x^{\alpha_{1}}, \phi(y)^{\alpha_{1}-\alpha_{0}} x^{\alpha_{0}}\right)\right)^{p}\right\|_{q / p, u} d y \\
& \geqslant\left\|\int_{0}^{\infty}\left(\min \left(x^{\alpha_{1}}, \phi(y)^{\alpha_{1}-\alpha_{0}} x^{\alpha_{0}}\right)\right)^{p} d y\right\|_{q / p, u} \\
& =\left\{\int_{0}^{\infty}\left[\int_{0}^{\infty}\left(\min \left(x^{\alpha_{1}}, \phi(y)^{\alpha_{1}-\alpha_{0}} x^{\alpha_{0}}\right)\right)^{p} d y\right]^{q / p} u(x) d x\right\}^{p / q} \\
& =\left\{\int_{0}^{\infty}\left[\int_{0}^{\phi^{-1}(x)} x^{p \alpha_{1}} d y+\int_{\phi^{-1}(x)}^{\infty} \phi(y)^{p\left(\alpha_{1}-\alpha_{0}\right)} x^{p \alpha_{0}} d y\right]^{q / p} u(x) d x\right\}^{p / q} \\
& =\left\{\int_{0}^{\infty}\left[\phi^{-1}(x) x^{p \alpha_{1}}+x^{p \alpha_{0}} \int_{\phi^{-1}(x)}^{\infty} \phi(y)^{p\left(\alpha_{1}-\alpha_{0}\right)} d y\right]^{q / p} u(x) d x\right\}^{p / q} \\
& =\left\{\int_{0}^{\infty}\left[x^{p \alpha_{0}} \int_{0}^{x} \phi^{-1}(s) d\left(s^{p\left(\alpha_{1}-\alpha_{0}\right)}\right)\right]^{q / p} u(x) d x\right\}^{p . q},
\end{aligned}
$$

since, by Lemma 1 (2),

$$
\int_{\phi^{-1}(x)}^{\infty} \phi(y)^{p\left(\alpha_{1}-\alpha_{0}\right)} d y=\int_{0}^{x} \phi^{-1}(s) d\left(s^{p\left(\alpha_{1}-\alpha_{0}\right)}\right)-\phi^{-1}(x) x^{p\left(\alpha_{1}-\alpha_{0}\right)} .
$$


Similarly, by Fubini's theorem and Lemma 1 (2),

$$
\begin{aligned}
\int_{0}^{\infty}\left\{\int_{0}^{\infty}\right. & {\left.\left[\min \left(x^{\alpha_{1}}, \phi(y)^{\alpha_{1}-\alpha_{0}} x^{\alpha_{0}}\right)\right]^{p} v(x) d x\right\} d y } \\
& =\int_{0}^{\infty}\left[\int_{0}^{\infty}\left(\min \left(x^{\alpha_{1}}, \phi(y)^{\alpha_{1}-\alpha_{0}} x^{\alpha_{0}}\right)\right)^{p} d y\right] v(x) d x \\
& =\int_{0}^{\infty}\left[\phi^{-1}(x) x^{p \alpha_{1}}+x^{p \alpha_{0}} \int_{\phi^{-1}(x)}^{\infty} \phi(y)^{p\left(\alpha_{1}-\alpha_{0}\right)} d y\right] v(x) d x \\
& =\int_{0}^{\infty}\left[x^{p \alpha_{0}} \int_{0}^{x} \phi^{-1}(s) d\left(s^{p\left(\alpha_{1}-\alpha_{0}\right)}\right)\right] v(x) d x .
\end{aligned}
$$

Putting the above inequalities and equalities together we obtain the crucial inequality

$$
\left\{\int_{0}^{\infty}\left[x^{p \alpha_{0}} \int_{0}^{x} \phi^{-1}(s) d\left(s^{p\left(\alpha_{1}-\alpha_{0}\right)}\right)\right]^{q / p} u(x) d x\right\}^{p / q} \leqslant D^{p} \int_{0}^{\infty}\left[x^{p \alpha_{0}} \int_{0}^{x} \phi^{-1}(s) d\left(s^{p\left(\alpha_{1}-\alpha_{0}\right)}\right)\right] v(x) d x .
$$

Now, if $g$ has the representation (6), then by taking

$$
\phi^{-1}(x)=m\left(x^{\alpha_{1}-\alpha_{0}}\right)\left[x^{-\alpha_{1}} g(x)\right]^{p-1}=\frac{x^{1-p\left(\alpha_{1}-\alpha_{0}\right)}}{p\left(\alpha_{1}-\alpha_{0}\right)} \frac{d}{d x}\left[x^{-p \alpha_{0}} g(x)^{p}\right]
$$

as a decreasing function, we obtain

$$
\int_{0}^{x} \phi^{-1}(s) d\left(s^{p\left(\alpha_{1}-\alpha_{0}\right)}\right)=x^{-p \alpha_{0}} g(x)^{p}-\lim _{s \rightarrow 0^{+}} s^{-p \alpha_{0}} g(s)^{p}=x^{-p \alpha_{0}} g(x)^{p},
$$

which we can substitute in (7) and get

$$
\left(\int_{0}^{\infty} g(x)^{q} u(x) d x\right)^{p / q} \leqslant D^{p} \int_{0}^{\infty} g(x)^{p} v(x) d x .
$$

Since $f \leqslant g \leqslant 2 f$ it follows that (4) holds and $C \leqslant 2 D$.

REMARK 3. The proof above shows that inequality (4) holds for every $f$ having the representation (6) if and only if (5) holds and that in this case $C=D$.

REMARK 4. Let $1 \leqslant p \leqslant q<\infty$. If we take $u(x)=x^{-\alpha q-1}$ and $v(x)=x^{-\alpha p-1}$ with $\alpha_{0}<\alpha<\alpha_{1}$, then $D=p^{1 / p} q^{-1 / q}\left(\left(\alpha_{1}-\alpha\right)^{-1}+\left(\alpha-\alpha_{0}\right)^{-1}\right)^{1 / q-1 / p}$.

Special choices of $\alpha_{0}, \alpha_{1}$ and $m$ can give some known results for decreasing functions, which have been proved previously, but not always with the best constant and not in the full range $0<p \leqslant q<\infty$ of the parameters $p$ and $q$.

Corollary 1. If $1 \leqslant p \leqslant q<\infty$, then

$$
\sup _{0 \leqslant f \downarrow} \frac{\left\|\int_{0}^{x} f\right\|_{q, u}}{\left\|\int_{0}^{x} f\right\|_{p, v}}=\sup _{t>0} \frac{\|\min (x, t)\|_{q, u}}{\|\min (x, t)\|_{p, v}} .
$$


In the above proof take $\alpha_{0}=0, \alpha_{1}=1$ and $m=f$ with $f$ a positive decreasing function. This result with the best constant but with some additional restrictions on $p$ and $q$ was proved in [8, Theorem 3.7]. Similarly we can get the result from [8, Theorem 4.3(a)].

COROLlary 2. If $1 \leqslant p \leqslant q<\infty$, then

$$
\sup _{0 \leqslant f \text { concave }} \frac{\|f\|_{q, u}}{\|f\|_{p, v}}=\sup _{t>0} \frac{\|\min (x, t)\|_{q, u}}{\|\min (x, t)\|_{p, v}} .
$$

Corollary 3. If $1 \leqslant p \leqslant q<\infty$, then

$$
\sup _{0 \leqslant f \downarrow} \frac{\left\|\frac{1}{x} \int_{0}^{x} f\right\|_{q, u}}{\left\|\frac{1}{x} \int_{0}^{x} f\right\|_{p, v}}=\sup _{t>0} \frac{\|\min (1, t / x)\|_{q, u}}{\|\min (1, t / x)\|_{p, v}} .
$$

In the proof of Theorem 1 take $\alpha_{0}=-1, \alpha_{1}=0$ and $m=f$, where $f$ is a positive decreasing function. This result without the best constant (only equivalence of the above expressions) was proved in a quite different way by Stepanov [17, Theorem 3.3].

Remark 5. Using the discretization method, Gol'dman, Heinig and Stepanov [7] established the equivalence of inequality (4) for $f \in Q^{0} \cap Q_{1}$ with inequality (5) in the case $0<p \leqslant q<\infty$, as well as the equivalence in the case $0<q<p<\infty$ with a certain complicated expression corresponding to (5). Our proof here (only the case $1 \leqslant p \leqslant q<\infty)$ is different and with a good control of the constants.

Acknowledgements. The author would like to thank the referee for information about the paper [7] and to Professor H. Heinig for sending me a preprint of it in May 1995.

\section{References}

1. J. Bergh, 'A converse inequality of Hölder type', Math. Z. 215 (1994) 205-208.

2. J. Bergh, V. Burenkov and L. E. Persson, 'Best constants in reversed Hardy's inequalities for quasimonotone functions', Acta Sci. Math. (Szeged) 59 (1994) 223-241.

3. J. BERGH and J. LÖFSTRÖM, Interpolation spaces (Springer, New York, 1976).

4. N. H. Bingham, C. M. Goldie and J. L. Teugels, Regular variation (Cambridge University Press, 1987).

5. L. Y. Chan, 'Some properties of asymptotic functions and their applications', Proc. Amer. Math. Soc. 72 (1978) 239-247.

6. Y. M. Chen, 'Theorems of asymptotic approximation', Math. Ann. 140 (1960) 360-407.

7. M. L. Gol'dman, H. Heinig and V. D. Stepanov, 'On the principle of duality in Lorentz spaces', Canad. J. Math. 48 (1996) 959-979.

8. H. Heinig and L. Maligranda, 'Weighted inequalities for monotone and concave functions', Studia Math. 116 (1995) 133-165.

9. H. Heinig and V. D. Stepanov, 'Weighted Hardy inequalities for increasing functions', Canad. J. Math. 45 (1993) 104-116.

10. L. Maligranda, Orlicz spaces and interpolation, Seminars in Mathematics 5, Universidade Estadual de Campinas, Campinas, SP, Brazil, 1989.

11. C. J. Neugebauter, 'Weighted norm inequalities for averaging operators of monotone functions', Publ. Mat. 35 (1991) 429-447.

12. J. PeČArIĆ and L. E. Persson, 'On Bergh's inequality for quasi-monotone functions', J. Math. Anal. Appl. 195 (1995) 393-400.

13. L. E. Persson, 'On a weak-type theorem with applications', Proc. London Math. Soc. 38 (1979) 295-308. 
14. E. SAWYER, 'Boundedness of classical operators on classical Lorentz spaces', Studia Math. 96 (1990) $145-158$.

15. E. Stein and G. Weiss, Introduction to Fourier analysis on Euclidean spaces (Princeton University Press, 1971).

16. V. D. StePAnOv, 'Weighted Hardy's inequality for nonincreasing functions', Trans. Amer. Math. Soc. 338 (1993) 173-186.

17. V. D. Stepanov, 'Integral operators on the cone of monotone functions', J. London Math. Soc. 48 (1993) 465-487.

18. A. C. ZAANEN, Linear analysis (Interscience, Amsterdam-Gröningen-New York, 1953).

Department of Mathematics

Luleå University of Technology

S-971 87 Luleå

Sweden

E-mail:1ech@sm.luth.se 